\title{
Preimage Attacks on Reduced Tiger and SHA-2
}

\author{
Takanori Isobe and Kyoji Shibutani \\ Sony Corporation \\ 1-7-1 Konan, Minato-ku, Tokyo 108-0075, Japan \\ \{Takanori.Isobe,Kyoji.Shibutani\}@jp.sony.com
}

\begin{abstract}
This paper shows new preimage attacks on reduced Tiger and SHA-2. Indesteege and Preneel presented a preimage attack on Tiger reduced to 13 rounds (out of 24 ) with a complexity of $2^{128.5}$. Our new preimage attack finds a one-block preimage of Tiger reduced to 16 rounds with a complexity of $2^{161}$. The proposed attack is based on meet-in-themiddle attacks. It seems difficult to find "independent words" of Tiger at first glance, since its key schedule function is much more complicated than that of MD4 or MD5. However, we developed techniques to find independent words efficiently by controlling its internal variables. Surprisingly, the similar techniques can be applied to SHA-2 including both SHA-256 and SHA-512. We present a one-block preimage attack on SHA-256 and SHA-512 reduced to 24 (out of 64 and 80) steps with a complexity of $2^{240}$ and $2^{480}$, respectively. To the best of our knowledge, our attack is the best known preimage attack on reduced-round Tiger and our preimage attack on reduced-step SHA-512 is the first result. Furthermore, our preimage attacks can also be extended to second preimage attacks directly, because our attacks can obtain random preimages from an arbitrary IV and an arbitrary target.
\end{abstract}

Keywords: hash function, preimage attack, second preimage attack, meet-in-the-middle, Tiger, SHA-256, SHA-512.

\section{Introduction}

Cryptographic hash functions play an important role in the modern cryptology. Many cryptographic protocols require a secure hash function which holds several security properties such as classical ones: collision resistance, preimage resistance and second preimage resistance. However, a lot of hash functions have been broken by collision attacks including the attacks on MD4 [3, MD5 [1] and SHA1 [12. These hash functions are considered to be broken in theory, but in practice many applications still use these hash functions because they do not require collision resistance. However, (second) preimage attacks are critical for many applications including integrity checks and encrypted password systems. Thus analyzing the security of the hash function with respect to (second) preimage resistance is important, even if the hash function is already broken by a collision attack. However, the preimage resistance of hash functions has not been studied well. 
Table 1. Summary of our results

\begin{tabular}{|c|c|c|c|}
\hline Target & $\begin{array}{c}\text { Attack } \\
\text { (first or second preimage) }\end{array}$ & $\begin{array}{c}\text { Attacked steps } \\
\text { (rounds) }\end{array}$ & Complexity \\
\hline Tiger (full 24 rounds) & first [4] & 13 & $2^{128.5}$ \\
\cline { 2 - 4 } & first (this paper) & 16 & $2^{161}$ \\
\cline { 2 - 4 } & second [4 & 13 & $2^{127.5}$ \\
\cline { 2 - 4 } & second (this paper) & 16 & $2^{160}$ \\
\hline \multirow{4}{*}{ SHA-256 (full 64 steps) } & first [10] & 36 & $2^{249}$ \\
\cline { 2 - 4 } & first (this paper) & 24 & $2^{240}$ \\
\cline { 2 - 4 } & second (this paper) & 24 & $2^{240}$ \\
\hline SHA-512 (full 80 steps) & first (this paper) & 24 & $2^{480}$ \\
\cline { 2 - 4 } & second (this paper) & 24 & $2^{480}$ \\
\hline
\end{tabular}

Tiger is a dedicated hash function producing a 192-bit hash value designed by Anderson and Biham in 1996 [2]. As a cryptanalysis of Tiger, at FSE 2006, Kelsey and Lucks proposed a collision attack on 17-round Tiger with a complexity of $2^{49}$ [5], where full-version Tiger has 24 rounds. They also proposed a pseudo-near collision attack on 20-round Tiger with a complexity of $2^{48}$. This attack was improved by Mendel et al. at INDOCRYPT 2006 [8]. They proposed a collision attack on 19-round Tiger with a complexity of $2^{62}$, and a pseudo-near collision attack on 22-round Tiger with a complexity of $2^{44}$. Later, they proposed a pseudo-near-collision attack of full-round (24-round) Tiger with a complexity of $2^{44}$, and a pseudo-collision (free-start-collision) attack on 23-round Tiger 9 . The above results are collision attacks and there is few evaluations of preimage resistance of Tiger. Indesteege and Preneel presented preimage attacks on reduced-round Tiger [4]. Their attack found a preimage of Tiger reduced to 13 rounds with a complexity of $2^{128.5}$.

In this paper, we introduce a preimage attack on reduced-round Tiger. The proposed attack is based on meet-in-the-middle attacks [1]. In this attack, we need to find independent words ("neutral words") in the first place. However, the techniques used for finding independent words of MD4 or MD5 cannot be applied to Tiger directly, since its key schedule function is much more complicated than that of MD4 or MD5. To overcome this problem, we developed new techniques to find independent words of Tiger efficiently by adjusting the internal variables. As a result, the proposed attack finds a preimage of Tiger reduced to 16 (out of 24) rounds with a complexity of about $2^{161}$. Surprisingly, our new approach can be applied to SHA-2 including both SHA-256 and SHA-512. We present a preimage attack on SHA-256 and SHA-512 reduced to 24 (out of 64 and 80) steps with a complexity of about $2^{240}$ and $2^{480}$, respectively. As far as we know, our attack is the best known preimage attack on reduced-round Tiger and our preimage attack on reduced-step SHA-512 is the first result. Furthermore, we show that our preimage attacks can also be extended to second preimage attacks directly and all of our attacks can obtain one-block preimages, because our preimage attacks can obtain random preimages from an arbitrary IV and an arbitrary target. These results are summarized in Table 1. 
This paper is organized as follows. Brief descriptions of Tiger, SHA-2 and the meet-in-the-middle approach are given in Section 2. A preimage attack on reduced-round Tiger and its extensions are shown in Section 3. In Section 4, we present a preimage attack on reduced-step SHA-2. Finally, we present conclusions in Section 5 .

\section{Preliminaries}

\subsection{Description of Tiger}

Tiger is an iterated hash function that compresses an arbitrary length message into a 192-bit hash value. An input message value is divided into 512-bit message blocks $\left(M^{(0)}, M^{(1)}, \ldots, M^{(t-1)}\right)$ by the padding process as well as the MD family. The compression function of Tiger shown in Fig. 1 generates a 192-bit output chaining value $H^{(i+1)}$ from a 512-bit message block $M^{(i)}$ and a 192-bit input chaining value $H^{(i)}$ where chaining values consist of three 64 -bit variables, $A_{j}^{(i)}$, $B_{j}^{(i)}$ and $C_{j}^{(i)}$. The initial chaining value $H^{(0)}=\left(A_{0}^{(0)}, B_{0}^{(0)}, C_{0}^{(0)}\right)$ is as follows:

$$
\begin{aligned}
& A_{0}^{(0)}=0 \mathrm{x} 0123456789 \mathrm{ABCDEF}, \\
& B_{0}^{(0)}=\text { 0xFEDCBA9876543210, } \\
& C_{0}^{(0)}=\text { 0xF096A5B4C3B2E187. }
\end{aligned}
$$

In the compression function, a 512-bit message block $M^{(i)}$ is divided into eight 64bit words $\left(X_{0}, X_{1}, \ldots, X_{7}\right)$. The compression function consists of three pass functions and between each of them there is a key schedule function. Since each pass function has eight round functions, the compression function consists of 24 round functions. The pass function is used for updating chaining values, and the key schedule function is used for updating message values. After the third pass function, the following feedforward process is executed to give outputs of the compression function with input chaining values and outputs of the third pass function,

$$
A_{24}^{\prime}=A_{0} \oplus A_{24}, B_{24}^{\prime}=B_{0}-B_{24}, C_{24}^{\prime}=C_{0}+C_{24},
$$

where $A_{i}, B_{i}$ and $C_{i}$ denote the $i$-th round chaining values, respectively, and $A_{24}^{\prime}, B_{24}^{\prime}$ and $C_{24}^{\prime}$ are outputs of the compression function.

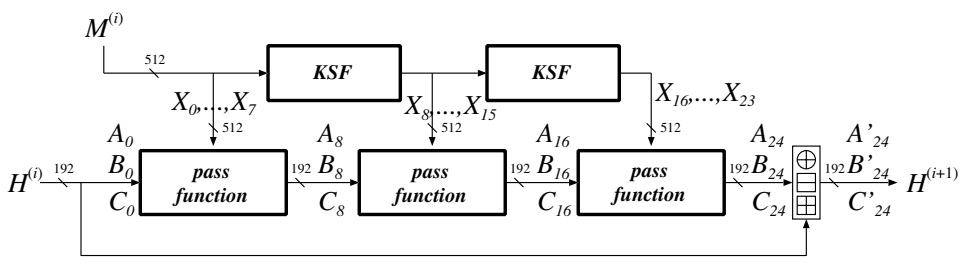

Fig. 1. Compression function $f$ of Tiger 
In each round of the pass function, chaining values $A_{i}, B_{i}$ and $C_{i}$ are updated by a message word $X_{i}$ as follows:

$$
\begin{aligned}
& B_{i+1}=C_{i} \oplus X_{i} \\
& C_{i+1}=A_{i}-\operatorname{even}\left(B_{i+1}\right), \\
& A_{i+1}=\left(B_{i}+\operatorname{odd}\left(B_{i+1}\right)\right) \times m u l,
\end{aligned}
$$

where $m u l$ is the constant value $\in\{5,7,9\}$ which is different in each pass function. The nonlinear functions even and odd are expressed as follows:

$$
\begin{aligned}
\operatorname{even}(W) & =T_{1}\left[w_{0}\right] \oplus T_{2}\left[w_{2}\right] \oplus T_{3}\left[w_{4}\right] \oplus T_{4}\left[w_{6}\right], \\
\operatorname{odd}(W) & =T_{4}\left[w_{1}\right] \oplus T_{3}\left[w_{3}\right] \oplus T_{2}\left[w_{5}\right] \oplus T_{1}\left[w_{7}\right],
\end{aligned}
$$

where 64 -bit value $W$ is split into eight bytes $\left\{w_{7}, w_{6}, \ldots, w_{0}\right\}$ with $w_{7}$ is the most significant byte and $T_{1}, \ldots, T_{4}$ are the S-boxes: $\{0,1\}^{8} \rightarrow\{0,1\}^{64}$. Figure 2 shows the round function of Tiger.

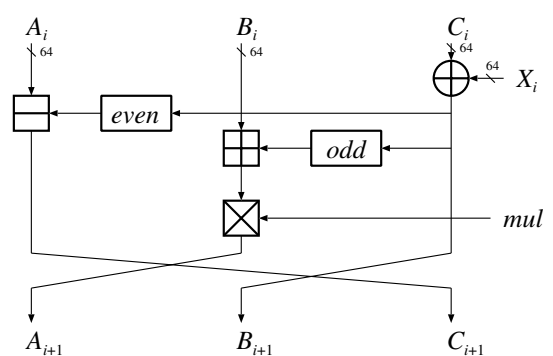

Fig. 2. Tiger round function

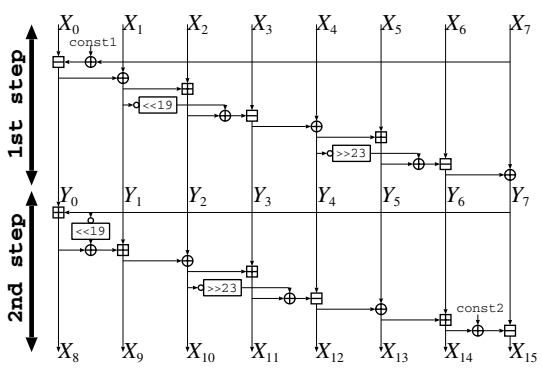

Fig. 3. Key schedule function

The key schedule function $(K S F)$ updates message values. In the first pass function, eight message words $X_{0}, \ldots, X_{7}$, which are identical to input message blocks of the compression function, are used for updating chaining values. Remaining two pass functions use sixteen message words which are generated by applying $K S F$ :

$$
\begin{aligned}
\left(X_{8}, \ldots, X_{15}\right) & =K S F\left(X_{0}, \ldots, X_{7}\right), \\
\left(X_{16}, \ldots, X_{23}\right) & =K S F\left(X_{8}, \ldots, X_{15}\right) .
\end{aligned}
$$

The function $K S F$ which updates the inputs $X_{0}, \ldots, X_{7}$ in two steps, is shown in Table 2. The first step shown in the left table generates internal variables $Y_{0}, \ldots, Y_{7}$ from inputs $X_{0}, \ldots, X_{7}$, and the second step shown in the right table calculates outputs $X_{8}, \ldots, X_{15}$ from internal variables $Y_{0}, . ., Y_{7}$, where const1 is 0xA5A5A5A5A5A5A5A5 and const2 is 0x0123456789ABCDEF. By using the same function, $X_{16}, \ldots, X_{23}$ are also derived from $X_{8}, \ldots, X_{15}$. Figure 3 shows the key schedule function of Tiger. 
Table 2. Algorithm of the key schedule function $K S F$

\begin{tabular}{|c|c|c|c|}
\hline$Y_{0}=X_{0}-\left(X_{7} \oplus\right.$ const 1$)$ & (8) & $X_{8}=Y_{0}+Y_{7}$ & $(16)$ \\
\hline$Y_{1}=X_{1} \oplus Y_{0}$ & $(9)$ & $X_{9}=Y_{1}-\left(X_{8} \oplus\left(\overline{Y_{7}} \ll 19\right)\right)$, & $(17)$ \\
\hline$Y_{2}=X_{2}+Y_{1}$ & $(10)$ & $X_{10}=Y_{2} \oplus X_{9}$ & $(18)$ \\
\hline$Y_{3}=X_{3}-\left(Y_{2} \oplus\left(\overline{Y_{1}} \ll 19\right)\right)$, & $(11)$ & $X_{11}=Y_{3}+X_{10}$ & (19) \\
\hline$Y_{4}=X_{4} \oplus Y_{3}$ & $(12)$ & $X_{12}=Y_{4}-\left(X_{11} \oplus\left(\overline{X_{10}} \gg 23\right)\right)$ & $(20)$ \\
\hline$Y_{5}=X_{5}+Y_{4}$ & $(13)$ & $X_{13}=Y_{5} \oplus X_{12}$ & $(21)$ \\
\hline$Y_{6}=X_{6}-\left(Y_{5} \oplus\left(\overline{Y_{4}} \gg 23\right)\right)$ & $(14)$ & $X_{14}=Y_{6}+X_{13}$ & $(22)$ \\
\hline$Y_{7}=X_{7} \oplus Y_{6}$ & $(15)$ & $X_{15}=Y_{7}-\left(X_{14} \oplus\right.$ const 2$)$ & $(23)$ \\
\hline
\end{tabular}

\subsection{Description of SHA-256}

We only show the structure of SHA-256, since SHA-512 is structurally very similar to SHA-256 except for the number of steps, word size and rotation values. The compression function of SHA-256 consists of a message expansion function and a state update function. The message expansion function expands 512-bit message block into 64 32-bit message words $W_{0}, \ldots, W_{63}$ as follows:

$$
W_{i}= \begin{cases}M_{i} & (0 \leq i<16), \\ \sigma_{1}\left(W_{i-2}\right)+W_{i-7}+\sigma_{0}\left(W_{i-15}\right)+W_{i-16} & (16 \leq i<64),\end{cases}
$$

where the functions $\sigma_{0}(X)$ and $\sigma_{1}(X)$ are given by

$$
\begin{aligned}
& \sigma_{0}(X)=(X \ggg 7) \oplus(X \ggg 18) \oplus(X \gg 3), \\
& \sigma_{1}(X)=(X \ggg 17) \oplus(X \ggg 19) \oplus(X \gg 10) .
\end{aligned}
$$

The state update function updates eight 32 -bit chaining values, $A, B, \ldots, G, H$ in 64 steps as follows:

$$
\begin{aligned}
T_{1} & =H_{i}+\Sigma_{1}\left(E_{i}\right)+C h\left(E_{i}, F_{i}, G_{i}\right)+K_{i}+W_{i}, \\
T_{2} & =\Sigma_{0}\left(A_{i}\right)+\operatorname{Maj}\left(A_{i}, B_{i}, C_{i}\right), \\
A_{i+1} & =T_{1}+T_{2}, \\
B_{i+1} & =A_{i} \\
C_{i+1} & =B_{i} \\
D_{i+1} & =C_{i} \\
E_{i+1} & =D_{i}+T_{1}, \\
F_{i+1} & =E_{i} \\
G_{i+1} & =F_{i}, \\
H_{i+1} & =G_{i},
\end{aligned}
$$


where $K_{i}$ is a step constant and the function $C h, M a j, \Sigma_{0}$ and $\Sigma_{1}$ are given as follows:

$$
\begin{aligned}
C h(X, Y, Z) & =X Y \oplus \bar{X} Z, \\
\operatorname{Maj}(X, Y, Z) & =X Y \oplus Y Z \oplus X Z, \\
\Sigma_{0}(X) & =(X \ggg 2) \oplus(X \ggg 13) \oplus(X \ggg 22), \\
\Sigma_{1}(X) & =(X \ggg 6) \oplus(X \ggg 11) \oplus(X \ggg 25) .
\end{aligned}
$$

After 64 step, a feedfoward process is executed with initial state variable by using word-wise addition modulo $2^{32}$.

\subsection{Meet-in-the-Middle Approach for Preimage Attack}

We assume that a compression function $F$ consists of a key scheduling function $(K S F)$ and a round/step function as shown in Fig. 4. The function $F$ has two inputs, an $n$-bit chaining variable $H$ and an $m$-bit message $M$, and outputs an $n$-bit chaining variable $G$. The function $K S F$ expands the message $M$, and provides them into the round/step function.

We consider a problem that given $H$ and $G$, find a message $M$ satisfying $G=F(H, M)$. This problem corresponds to the preimage attack on the compression function with a fixed input chaining variable. In this model, a feedforward function does not affect the attack complexity, since the targets $H$ and $G$ are arbitrary values. If we obtain a preimage from arbitrary values of $H$ and $G$, we can also compute a preimage from $H$ and $H \oplus G$ instead of $G$.

In the meet-in-the-middle preimage attack, we first divide the round function into two parts: the forward process $(F P)$ and the backward process $(B P)$ so that each process can compute an $\ell$-bit meet point $S$ independently. We also need independent words $X$ and $Y$ in $K S F$ to compute $S$ independently. The meet point $S$ can be determined from $F P$ and $B P$ independently such that $S=F P(H, X)$ and $S=B P(G, Y)$.

If there are such two processes $F P$ and $B P$, and independent words $X$ and $Y$, we can obtain a message $M$ satisfying $S$ with a complexity of $2^{\ell / 2} F$ evaluations, assuming that $F P$ and $B P$ are random ones, and the computation cost of $B P$ is almost same as that of inverting function of $B P$. Since remaining internal state value

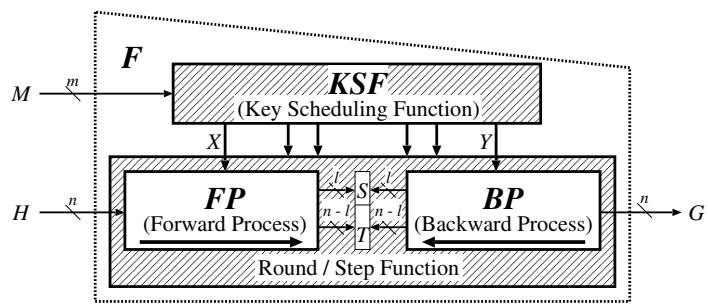

Fig. 4. Meet-in-the-middle approach 
$T$ is $(n-\ell)$ bits, the desired $M$ can be obtained with a complexity of $2^{n-\ell / 2}(=$ $\left.2^{n-\ell+\ell / 2}\right)$. Therefore, if $F P$ and $B P$ up to the meet point $S$ can be calculated independently, a preimage attack can succeed with a complexity of $2^{n-\ell / 2}$. This type of preimage attacks on MD4 and MD5 was presented by Aoki and Sasaki 1 .

In general, it is difficult to find such independent words in a complicated $K S F$. We developed new techniques to construct independent transforms in $K S F$ by controlling internal variabes to obtain independent words.

\section{Preimage Attack on Reduced-Round Tiger}

In this section, we propose a preimage attack on 16-round Tiger with a complexity of $2^{161}$. This variant shown in Fig. 5 consists of two pass functions and one key schedule function. First, we show properties of Tiger which are used for applying the meet-in-the-middle attack. Next, we show how to apply the meetin-the-middle attack to Tiger, and then introduce the algorithm of our attack. Finally, we evaluate the required complexity and memory of our attack.

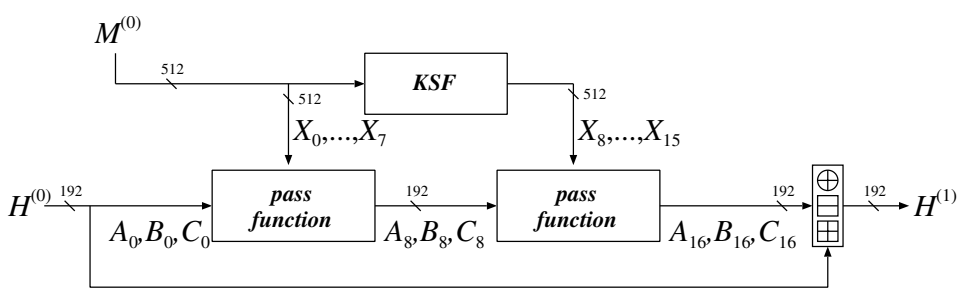

Fig. 5. Reduced-round Tiger (2-pass $=16$-round)

\subsection{Properties of Tiger}

We show five properties of Tiger, which enable us to apply the meet-in-themiddle attack.

Property 1: The pass function is easily invertible.

Property 1 can be obtained from the design of the round function. From Eq. (11) to Eq. (3) $, A_{i}, B_{i}$, and $C_{i}$ can be determined from $A_{i+1}, B_{i+1}, C_{i+1}$ and $X_{i}$. The computation cost is almost same as the cost of calculating $A_{i+1}, B_{i+1}$ and $C_{i+1}$ from $A_{i}, B_{i}, C_{i}$ and $X_{i}$. Since the round function is invertible, we can construct the inverse pass function.

Property 2: In the inverse pass function, the particular message words are independent of particular state value.

The detail of the Property 2 is that once $X_{i}, A_{i+3} B_{i+3}$ and $C_{i+3}$ are fixed, then $C_{i}, B_{i+1}, A_{i+2}$ and $B_{i+2}$ can be determined from Eq. (1) to Eq. (3) independently of $X_{i+1}$ and $X_{i+2}$. Thus the property 2 implies that $X_{i+1}$ and $X_{i+2}$ are independent of $C_{i}$ in the inverse pass function. 
Property 3: In the round function, $C_{i+1}$ is independent of odd bytes of $X_{i}$.

The property 3 can be obtained from the property of the non-linear function even.

Property 4: The key schedule function KSF is easily invertible.

The property 4 implies that we can build the inverse key schedule function $K S F^{-1}$. Moreover, the computation cost of $K S F^{-1}$ is almost the same as that of $K S F$.

Property 5: In the inverse key schedule function $K S F^{-1}$, if input values are chosen appropriately, there are two independent transforms.

The property 5 is one of the most important properties for our attack. In the next section, we show this in detail.

\subsection{How to Obtain Two Independent Transforms in the $K S^{-1}$}

Since any input word of $K S F^{-1}$ affects all output words of $K S F^{-1}$, it appears that there is no independent transform in the $K S F^{-1}$ at first glance.

However, we analyzed the relation among the inputs and the outputs of $K S F^{-1}$ deeply, and then found a technique to construct two independent transforms in the $K S F^{-1}$ by choosing inputs carefully and controlling internal variables. Specifically, we can show that a change of input word $X_{8}$ only affects output words $X_{0}, X_{1}, X_{2}$ and $X_{3}$, and also modifications of $X_{13}, X_{14}$ and $X_{15}$ only affect $X_{5}$ and $X_{6}$ if these input words are chosen properly. We present the relation among inputs, outputs and internal variables of $K S F^{-1}$ and then show how to build independent transforms in the $K S F^{-1}$.

As shown in Fig. 6] changes of inputs $X_{13}, X_{14}$ and $X_{15}$ only propagate internal variables $Y_{0}, Y_{1}, Y_{5}, Y_{6}$ and $Y_{7}$. If internal variables $Y_{6}$ and $Y_{7}$ are fixed

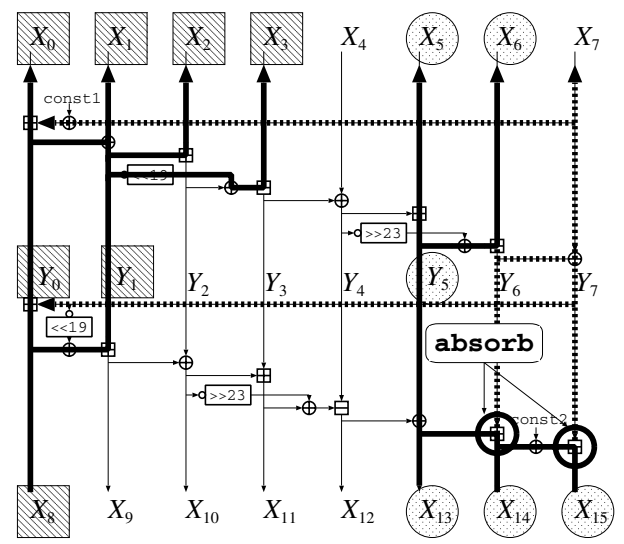

Fig. 6. Relation among inputs and outputs of $K S F^{-1}$ 
even when $X_{13}, X_{14}$ and $X_{15}$ are changed, it can be considered that an internal variable $Y_{0}, Y_{1}$ and an output $X_{7}$ are independent of changes of $X_{13}, X_{14}$ and $X_{15}$. From Eq. (22) and (23), $Y_{6}$ and $Y_{7}$ can be fixed to arbitrary values by choosing $X_{13}, X_{14}$ and $X_{15}$ satisfying the following formulae:

$$
\begin{aligned}
& X_{14}=Y_{6}+X_{13}, \\
& X_{15}=Y_{7}-\left(X_{14} \oplus \text { const } 2\right) .
\end{aligned}
$$

Therefore modifications of inputs $X_{13}, X_{14}$ and $X_{15}$ only propagate $X_{5}$ and $X_{6}$ by selecting these input values appropriately. In addition, a modification of $X_{8}$ only affects $X_{0}, \ldots, X_{3}$.

As a result, we obtain two independent transforms in $K S F^{-1}$ by choosing $X_{13}, X_{14}$ and $X_{15}$ properly, since in this case a change of $X_{8}$ only affects $X_{0}, \ldots$, $X_{3}$, and changes of $X_{13}, X_{14}$ and $X_{15}$ only propagate $X_{5}$ and $X_{6}$.

\subsection{Applying Meet-in-the-Middle Attack to Reduced-Round Tiger}

We show the method for applying the meet-in-the-middle attack to Tiger by using above five properties. We define the meet point as 64 -bit $C_{6}$, the process 1 as rounds 1 to 6 , and the process 2 as rounds 7 to 16 .

In the process 2 , intermediate values $A_{9}, B_{9}$ and $C_{9}$ can be calculated from $A_{16}, B_{16}, C_{16}$ and message words $X_{9}$ to $X_{15}$, since Tiger without the feedforward function is easily invertible. From the property $2, C_{6}$ can be determined from $A_{8}, B_{8}$ and $X_{6}$. It is also observed that $A_{8}$ and $B_{8}$ are independent of $X_{8}$, because these values are calculated from $A_{9}, B_{9}$ and $C_{9}$. From the property $5, X_{8}$ does not affect $X_{6}$. Therefore, $C_{6}$, the output of the process 2 , can be determined from $X_{6}, X_{9}$ to $X_{15}, A_{16}, B_{16}$ and $C_{16}$.

In the process 1 , the output $C_{6}$ can be calculated from $X_{0}$ to $X_{5}, A_{0}, B_{0}$ and $C_{0}$. If some changes of the message words used in each process do not affect the message words used in the other process, $C_{6}$ can be determined independently in each process.

The message words $X_{0}$ to $X_{4}$ are independent of changes of $X_{6}$ and $X_{13}$ to $X_{15}$, if $X_{9}$ to $X_{12}$ are fixed and $X_{13}$ to $X_{15}$ are calculated as illustrated in the section 3.2. Although changes of $X_{13}, X_{14}$ and $X_{15}$ propagate $X_{5}$, from the property $3, C_{6}$ in the process 1 is not affected by changes of odd bytes of $X_{5}$. Therefore, if even bytes of $X_{5}$ are fixed, $C_{6}$ in the process 1 can be determined independently from a change of $X_{5}$.

We show that the even bytes of $X_{5}$ can be fixed by choosing $X_{11}, X_{12}$ and $X_{13}$ properly. From Eq. (21), $Y_{5}$ is identical to $X_{13}$ when $X_{12}$ equals zero, and from Eq. (13), $X_{5}$ is identical to $Y_{5}$ when $Y_{4}$ equals zero. Thus $X_{5}$ is identical to $X_{13}$ when both $X_{12}$ and $Y_{4}$ are zero. Consequently, if the even bytes of $X_{13}$ are fixed, and $X_{12}$ and $Y_{4}$ equal zero, the even bytes of $X_{5}$ can be fixed. $Y_{4}$ can be fixed to zero by choosing $X_{11}$ as $X_{11} \leftarrow \overline{X_{10}} \gg 23$. Therefore, if the following conditions are satisfied, $C_{6}$ in the process 1 can be independent of changes of $X_{13}, X_{14}$ and $X_{15}$. 


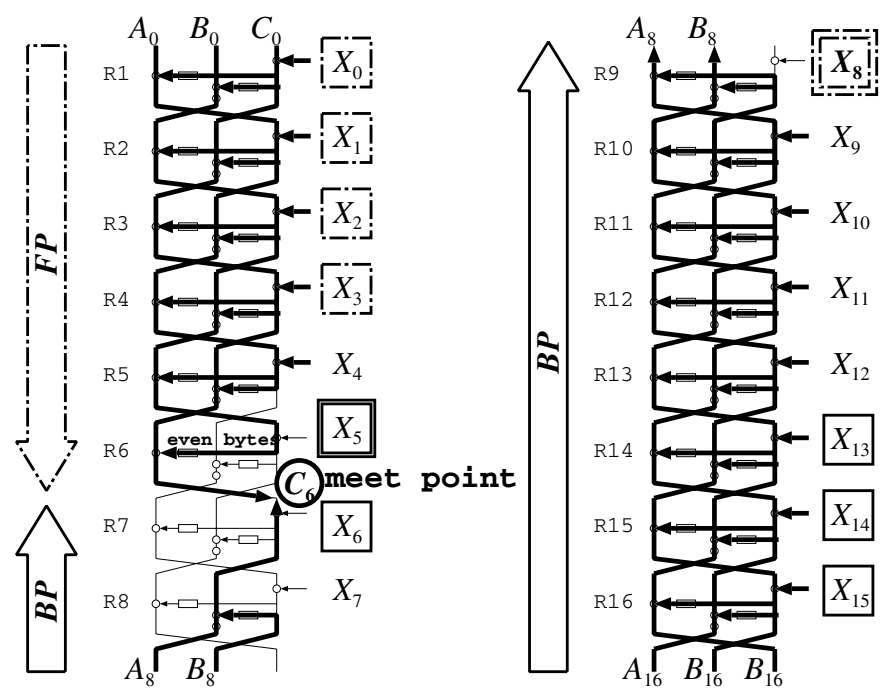

Fig. 7. Meet-in-the-middle attack on 16-round Tiger

$-X_{9}$ and $X_{10}$ are fixed arbitrarily,

$-X_{11}=\overline{X_{10}} \gg 23, X_{12}=0$,

- $X_{13}, X_{14}$ and $X_{15}$ are chosen properly.

By choosing inputs of the inverse pass function satisfying the above conditions, we can execute the process 1 and the process 2 independently. Specifically, if only $X_{13}, X_{14}$ and $X_{15}$ are treated as variables in the process 2 , then the process 2 can be executed independently from the process 1 . Similarly, if only $X_{8}$ is treated as a variable in the process 1 , then the process 1 is independent of the process 2 , as long as $X_{8}$ to $X_{15}$ satisfy the above conditions. These results are shown in Fig. 7 .

\section{4 (Second) Preimage Attack on 16-Round Tiger Compression Function}

We present the whole algorithm of the (second) preimage attack on the compression function of Tiger reduced to 16 rounds. The attack consists of three phases: preparation, first and second phase.

The preparation phase sets $X_{i}(i \in\{4,7,9,10,11,12\}), Y_{i}(i \in\{2,3,4,6,7\})$ and even bytes of $X_{13}$ as follows:

\section{Preparation}

1: Let $A_{16}^{\prime}, B_{16}^{\prime}$ and $C_{16}^{\prime}$ be given targets. Choose $A_{0}, B_{0}$ and $C_{0}$ arbitrarily, and set $A_{16}, B_{16}$ and $C_{16}$ as follows:

$$
A_{16} \leftarrow A_{0} \oplus A_{16}^{\prime}, B_{16} \leftarrow B_{0}-B_{16}^{\prime}, C_{16} \leftarrow C_{16}^{\prime}-C_{0} .
$$


2: Choose $X_{9}, X_{10}, Y_{6}, Y_{7}$ and even bytes of $X_{13}$ arbitrarily, set $X_{12}$ and $Y_{4}$ to zero, and set $X_{7}, X_{11}, Y_{2}, Y_{3}$ and $X_{4}$ as follows:

$$
X_{7} \leftarrow Y_{6} \oplus Y_{7}, X_{11} \leftarrow \overline{X_{10}} \gg 23, Y_{2} \leftarrow X_{9} \oplus X_{10}, Y_{3} \leftarrow X_{11}-X_{10}, X_{4} \leftarrow Y_{3} .
$$

The first phase makes a table of $\left(C_{6}\right.$, odd bytes of $\left.X_{13}\right)$ pairs in the process 2 as follows:

\section{First Phase}

1: Choose odd bytes of $X_{13}$ randomly.

2: Set $X_{5}, X_{6}, X_{14}$ and $X_{15}$ as follows:

$$
X_{5} \leftarrow X_{13}, X_{6} \leftarrow Y_{6}+X_{13}, X_{14} \leftarrow Y_{6}+X_{13}, X_{15} \leftarrow Y_{7}-\left(\left(Y_{6}+X_{13}\right) \oplus \text { const } 2\right) .
$$

3: Compute $C_{6}$ from $A_{16}, B_{16}, C_{16}, X_{6}$ and $X_{9}$ to $X_{15}$.

4: Place a pair $\left(C_{6}\right.$, odd bytes of $\left.X_{13}\right)$ into a table.

5: If all $2^{32}$ possibilities of odd bytes of $X_{13}$ have been checked, terminate this phase. Otherwise, set another value, which has not been set yet, to odd bytes of $X_{13}$ and return to the step 2 .

The second phase finds the desired message values $X_{0}$ to $X_{15}$ in the process 1 by using the table as follows:

\section{Second Phase}

1: Choose $X_{8}$ randomly.

2: Set $Y_{0}, Y_{1}, X_{0}, X_{1}, X_{2}$ and $X_{3}$ as follows:

$$
\begin{aligned}
& Y_{0} \leftarrow X_{8}-X_{7}, \\
& Y_{1} \leftarrow X_{9}+\left(X_{8} \oplus\left(\overline{Y_{7}} \ll 19\right)\right), \\
& X_{0} \leftarrow Y_{0}+\left(X_{7} \oplus \text { const } 1\right), \\
& X_{1} \leftarrow Y_{0} \oplus Y_{1}, \\
& X_{2} \leftarrow Y_{2}-Y_{1}, \\
& X_{3} \leftarrow Y_{3}+\left(Y_{2} \oplus\left(\overline{Y_{1}} \ll 19\right)\right) .
\end{aligned}
$$

3: Compute $C_{6}$ from $X_{0}$ to $X_{4}$, even bytes of $X_{5}, A_{0}, B_{0}$ and $C_{0}$.

4: Check whether this $C_{6}$ is in the table generated in the first phase. If $C_{6}$ is in the table, the corresponding $X_{0}$ to $X_{7}$ are a preimage for the compression function of the target $A_{16}^{\prime}, B_{16}^{\prime}, C_{16}^{\prime}$ and successfully terminates the attack. Otherwise, set another value, which has not been set yet, to $X_{8}$ and return to the step 2 .

By repeating the second phase about $2^{32}$ times for different choices of $X_{8}$, we expect to obtain a matched $C_{6}$. The complexity of the above algorithm is $2^{32}\left(=2^{32} \cdot \frac{6}{16}+2^{32} \cdot \frac{10}{16}\right)$ compression function evaluations, and success probability is about $2^{-128}$. By executing the above algorithm $2^{128}$ times with different fixed 
values, we can obtain a preimage of the compression function. In the preparation phase, $A_{0}, B_{0}, C_{0}, X_{9}, X_{10}, Y_{6}, Y_{7}$ and even bytes of $X_{13}$ can be chosen arbitrarily. In other words, this attack can use these values as free words. These free words are enough for searching $2^{128}$ space. Accordingly, the complexity of the preimage attack on the compression function is $2^{160}\left(=2^{32} \cdot 2^{128}\right)$. Also, this algorithm requires $2^{32} 96$-bit or $2^{35.6}$ bytes memory.

\subsection{One-Block (Second) Preimage Attack on 16-Round Tiger}

The preimage attack on the compression function can be extended to the oneblock preimage attack on 16-round Tiger hash function. For extending the attack, $A_{0}, B_{0}, C_{0}$ are fixed to the IV words, the padding word $X_{7}$ is fixed to 447 encoded in 64-bit string, and the remaining 224 bits are used as free bits in the preparation phase. Although our attack cannot deal with another padding word $X_{6}$, the attack still works when the least significant bit of $X_{6}$ equals one.

Hence, the success probability of the attack on the hash function is half of that of the attack on the compression function. The total complexity of the one-block preimage attack on 16-round Tiger hash function is $2^{161}$ compression function computations.

This preimage attack can also be extended to the one-block second preimage attack directly. Our second preimage attack obtains a one-block preimage with the complexity of $2^{161}$. Moreover, the complexity of our second preimage attack can be reduced by using the technique given in 4. In this case, the second preimage attack obtains the preimage which consists of at least two message blocks with a complexity of $2^{160}$.

\section{Preimage Attack on Reduced-Round SHA-2}

We apply our techniques to SHA-2 including both SHA-256 and SHA-512 in straightforward and present a preimage attack on SHA-2 reduced to 24 (out of 64 and 80, respectively) steps. We first check the properties of SHA-2, then introduce the algorithm of the preimage attack on 24-step SHA-2.

\subsection{Properties of 24-Step SHA-2}

We first check whether SHA-2 has similar properties of Tiger. The pass function of Tiger corresponds to the 16-step state update function of SHA-2, and the key schedule function of Tiger corresponds to the 16-step message expansion function of SHA-2. Since the state update function and the message expansion function of SHA-2 are easily invertible, the compression function of SHA-2 without the feedforward function is also invertible.

In the inverse state update function, $A_{18}, B_{18}, \ldots, H_{18}$ are determined from $A_{24}, B_{24}, \ldots, H_{24}$ and $W_{18}$ to $W_{23}$, and $A_{11}$ only depends on $A_{18}, \ldots, H_{18}$. Thus $A_{11}$ is independent of $W_{11}$ to $W_{17}$ when $A_{18}, \ldots, H_{18}$ and $W_{18}$ to $W_{23}$ are fixed. It corresponds to the property 2 of Tiger.

Then we check whether there are independent transforms in the inverse message expansion function of SHA-2. It corresponds to the property 5 of Tiger. For 


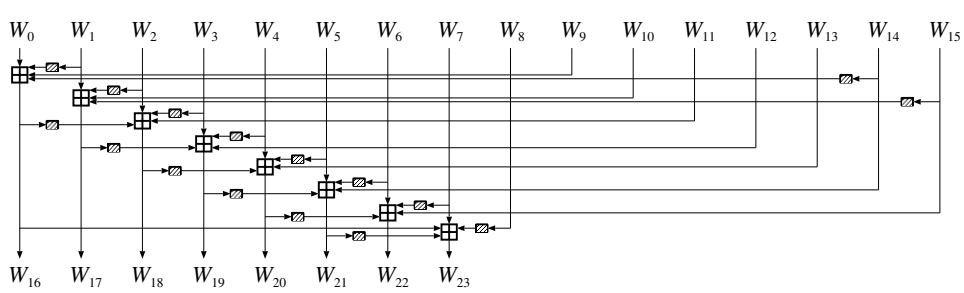

Fig. 8. Message expansion function of 24-step SHA-2

the 24-step SHA-2, 16 message words $W_{0}$ to $W_{15}$ used in the first 16 steps are identical to input message blocks of the compression function, and 8 message words $W_{16}$ to $W_{23}$ used in the remaining eight steps are derived from $W_{0}$ to $W_{15}$ by the message expansion function shown in Fig. 8. Table 3 shows the relation among message words in the message expansion function. For example, $W_{16}$ is determined from $W_{14}, W_{9}, W_{1}$ and $W_{0}$. By using these relation and techniques introduced in previous sections, we can configure two independent transforms in the message expansion function of SHA-2.

We show that, in the inverse message expansion function of 24-step SHA-2, i) a change of $W_{17}$ only affects $W_{0}, W_{1}, W_{3}$ and $W_{11}$, and ii) $W_{19}, W_{21}$ and $W_{23}$ only affect $W_{12}$ by using the message modification techniques. In Tab. 3, asterisked values are variables of $\mathrm{i}$ ), and underlined values are variables of ii).

First, we consider the influence of $W_{23}$. Though $W_{23}$ affects $W_{7}, W_{8}, W_{16}$ and $W_{21}$, this influence can be absorbed by modifying $W_{21} \rightarrow W_{19} \rightarrow W_{12}$. Consequently, we obtain a result that $W_{19}, W_{21}$ and $W_{23}$ only affect $W_{12}$ by choosing these values properly, since $W_{12}$ does not affect any other values in the inverse message expansion function.

Similarly, we consider the influence of $W_{17}$ in the inverse message expansion function. $W_{17}$ affects $W_{1}, W_{2}, W_{10}$ and $W_{15}$. This influence can be absorbed by modifying $W_{1} \rightarrow W_{0}$. $W_{17}$ is also used for generating $W_{19}$. In order to cancel this influence, $W_{3} \rightarrow W_{11}$ are also modified. As a result, we obtain a result that $W_{17}$ only affects $W_{0}, W_{1}, W_{3}$ and $W_{11}$ by choosing these values appropriately.

Table 3. Relation among message values $W_{16}$ to $W_{23}$

\begin{tabular}{|c|c|}
\hline computed value & values for computing \\
\hline$W_{16}$ & $W_{14}, W_{9}, W_{1} *, W_{0} *$ \\
\hline$W_{17} *$ & $W_{15}, W_{10}, W_{2}, W_{1} *$ \\
\hline$W_{18}$ & $W_{16}, W_{11} *, W_{3} *, W_{2}$ \\
\hline$\underline{W_{19}}$ & $W_{17} *, W_{12}, W_{4}, W_{3} *$ \\
\hline$W_{20}$ & $W_{18}, W_{13}, W_{5}, W_{4}$ \\
\hline$\underline{W_{21}}$ & $\underline{W_{19}}, W_{14}, W_{6}, W_{5}$ \\
\hline$W_{22}$ & $\underline{W_{20}, W_{15}, W_{7}, W_{6}}$ \\
\hline$\underline{W_{23}}$ & $\underline{W_{21}}, W_{16}, W_{8}, W_{7}$ \\
\hline
\end{tabular}



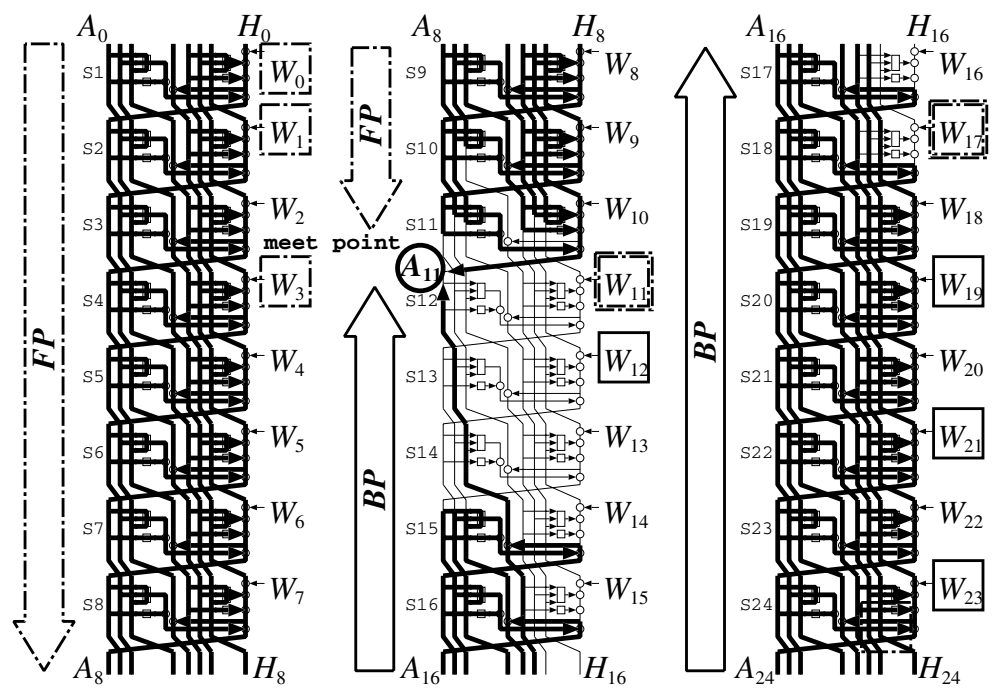

Fig. 9. Meet-in-the-middle attack on 24-step SHA-2

\section{2 (Second) Preimage Attack on 24-Step SHA-256 Compression Function}

As shown in Fig. 9, we define the meet point as 32-bit $A_{11}$, the process 1 as steps 1 to 11 , and the process 2 as steps 12 to 24 . In the process $1, A_{11}$ can be derived from $A_{0}, \ldots, H_{0}$ and $W_{0}$ to $W_{10}$. Similarly, in the process $2, A_{11}$ can be determined from $A_{24}, \ldots, H_{24}$ and $W_{18}$ to $W_{23}$. Since the process 1 and process 2 are independent of each other for $A_{11}$ by using the above properties of SHA-2, we apply the meet-in-the-middle attack to SHA-2 as follows:

\section{Preparation}

1: Let $A_{24}^{\prime}, \ldots, H_{24}^{\prime}$ be given targets. Choose $A_{0}, \ldots, H_{0}$ arbitrarily, and compute $A_{24}, \ldots, H_{24}$ by the feedforward function.

2: Choose 32 -bit value CON and $W_{i}(i \in\{2,4,5,6,7,8,9,10,13,14,15,16,18\})$ arbitrarily, and then calculate $W_{20}$ and $W_{22}$.

\section{First Phase}

1: Choose $W_{23}$ randomly.

2: Determine $W_{21}, W_{19}$ and $W_{12}$ as follow: 1 :

$$
\begin{aligned}
& W_{21} \leftarrow \sigma_{1}^{-1}\left(W_{23}-W_{16}-\sigma_{0}\left(W_{8}\right)-W_{7}\right), \\
& W_{19} \leftarrow \sigma_{1}^{-1}\left(W_{21}-W_{14}-\sigma_{0}\left(W_{6}\right)-W_{5}\right), \\
& W_{12} \leftarrow W_{19}-\text { CON. }
\end{aligned}
$$

\footnotetext{
${ }^{1}$ The method how to calculate $\sigma_{1}^{-1}$ is illustrated in the appendix.
} 
3: Compute $A_{11}$ from $A_{24}, \ldots, H_{24}$ and $W_{18}$ to $W_{23}$.

4: Place a pair $\left(A_{11}, W_{23}\right)$ into a table.

5: If $2^{16}$ pairs of $\left(A_{11}, W_{23}\right)$ have been listed in the table, terminate this algorithm. Otherwise, set another value, which has not been set yet, to $W_{23}$ and return to the step 2 .

\section{Second Phase}

1: Choose $W_{17}$ randomly.

2: Determine $W_{0}, W_{1}, W_{3}$ and $W_{11}$ as follows:

$$
\begin{aligned}
W_{1} & \leftarrow W_{17}-\sigma_{1}\left(W_{15}\right)-W_{10}-\sigma_{0}\left(W_{2}\right), \\
W_{0} & \leftarrow W_{16}-\sigma_{1}\left(W_{14}\right)-W_{9}-\sigma_{0}\left(W_{1}\right), \\
W_{3} & \leftarrow \mathrm{CON}-\sigma_{1}\left(W_{17}\right)-\sigma_{0}\left(W_{4}\right), \\
W_{11} & \leftarrow W_{18}-\sigma_{1}\left(W_{16}\right)-\sigma_{0}\left(W_{3}\right)-W_{2} .
\end{aligned}
$$

3: Compute $A_{11}$ from $A_{0}, \ldots, H_{0}$ and $W_{0}$ to $W_{10}$.

4: Check whether this $A_{11}$ is in the table generated in the first phase. If $A_{11}$ is in the table, the corresponding $W_{0}$ to $W_{23}$ is a preimage of the compression function of the target $A_{24}^{\prime}, \ldots, H_{24}^{\prime}$ and successfully terminates the attack. Otherwise, set another value, which has not been set yet, to $W_{17}$ and return to the step 2.

By repeating the second phase about $2^{16}$ times for different $W_{17}$, we expect to obtain a matched $A_{11}$. The complexity of the preimage attack on the compression function is $2^{240}\left(=2^{256-32 / 2}\right)$ compression function evaluations. The required memory is $2^{16} 64$-bit or $2^{19}$ bytes. In this attack, the words $A_{0}, \ldots, H_{0}$, CON and $W_{i}(i \in\{2,4,5,6,7,8,9,10,13,14,15,16,18\})$ can be used as free words. The total free words are 22 words or 704 bits.

\subsection{One-Block (Second) Preimage Attack on 24-Step SHA-2 Hash Function}

The preimage attack on the compression function can be extended to the (second) preimage attack on the hash function directly, since our preimage attack can obtain random preimages from an arbitrary IV and an arbitrary target, and can deal with the padding words $W_{14}$ and $W_{15}$. Thus the complexities of the preimage attack and the second preimage attack on 24-step SHA-256 are $2^{240}$. Furthermore, this attack can also be extended to the (second) preimage attack on 24-step SHA-512. The complexities of the (second) preimage attack on 24-step SHA-512 are $2^{480}\left(=2^{512-64 / 2}\right)$.

\section{Conclusion}

In this paper, we have shown preimage attacks on reduced-round Tiger, reducedstep SHA-256 and reduced-step SHA-512. The proposed attacks are based on 
meet-in-the-middle attack. We developed new techniques to find "independent words" of the compression functions. In the attack on reduced-round Tiger, we found the "independent transforms" in the message schedule function by adjusting the internal variables, then we presented there are independent words in the compression function of Tiger. In the attack on reduced-round SHA-2, we found the "independent transforms" in the message expansion function by modifying the messages, then we showed that there are independent words in the compression function of SHA-2.

Our preimage attack can find a preimage of 16-step Tiger, 24-step SHA-256 and 24-step SHA-512 with a complexity of $2^{161}, 2^{240}$ and $2^{480}$, respectively. These preimage attacks can be extended to second preimage attacks with the almost same complexities. Moreover, our (second) preimage attacks can find a one-block preimage, since it can obtain random preimages from an arbitrary IV an arbitrary target, and can also deal with the padding words.

Acknowledgments. The authors thank to the anonymous referees for their valuable comments.

\section{References}

1. Aoki, K., Sasaki, Y.: Preimage attacks on one-block MD4, 63-step MD5 and more. In: Avanzi, R., Keliher, L., Sica, F. (eds.) SAC 2008. LNCS, pp. 82-98. Springer, Heidelberg (2008)

2. Anderson, R., Biham, E.: Tiger: A fast new hash function. In: Gollmann, D. (ed.) FSE 1996. LNCS, vol. 1039, pp. 89-97. Springer, Heidelberg (1996)

3. Dobbertin, H.: Cryptanalysis of MD4. In: Gollmann, D. (ed.) FSE 1996. LNCS, vol. 1039, pp. 53-69. Springer, Heidelberg (1996)

4. Indesteege, S., Preneel, B.: Preimages for reduced-round Tiger. In: Lucks, S., Sadeghi, A.-R., Wolf, C. (eds.) WEWoRC 2007. LNCS, vol. 4945, pp. 90-99. Springer, Heidelberg (2008)

5. Kelsey, J., Lucks, S.: Collisions and near-collisions for reduced-round Tiger. In: Robshaw, M.J.B. (ed.) FSE 2006. LNCS, vol. 4047, pp. 111-125. Springer, Heidelberg (2006)

6. Leurent, G.: MD4 is not one-way. In: Nyberg, K. (ed.) FSE 2008. LNCS, vol. 5086, pp. 412-428. Springer, Heidelberg (2008)

7. Mendel, F., Pramstaller, N., Rechberger, C.: A (second) preimage attack on the GOST hash function. In: Nyberg, K. (ed.) FSE 2008. LNCS, vol. 5086, pp. 224-234. Springer, Heidelberg (2008)

8. Mendel, F., Preneel, B., Rijmen, V., Yoshida, H., Watanabe, D.: Update on Tiger. In: Barua, R., Lange, T. (eds.) INDOCRYPT 2006. LNCS, vol. 4329, pp. 63-79. Springer, Heidelberg (2006)

9. Mendel, F., Rijmen, V.: Cryptanalysis of the Tiger hash function. In: Kurosawa, K. (ed.) ASIACRYPT 2007. LNCS, vol. 4833, pp. 536-550. Springer, Heidelberg (2007)

10. Sasaki, Y., Aoki, K.: Preimage Attacks on MD, HAVAL, SHA, and Others. In: Rump session at CRYPTO 2008 (2008)

11. Wang, X., Yu, H.: How to break MD5 and other hash functions. In: Cramer, R. (ed.) EUROCRYPT 2005. LNCS, vol. 3494, pp. 19-35. Springer, Heidelberg (2005)

12. Wang, X., Yin, Y.L., Yu, H.: Finding collisions in the full SHA-1. In: Shoup, V. (ed.) CRYPTO 2005. LNCS, vol. 3621, pp. 17-36. Springer, Heidelberg (2005) 


\section{Appendix A}

Here, we show how to calculate the inverse function $\sigma_{1}^{-1}$. Let $\left(x_{31}, \ldots, x_{0}\right)$ and $\left(y_{31}, \ldots, y_{0}\right)$ be outputs and inputs of $\sigma_{1}^{-1}$ respectively, where $x_{i}, y_{i} \in\{0,1\}$, and $x_{31}$ and $y_{31}$ are the most significant bit. The inverse function $\sigma_{1}^{-1}$ is calculated as follows:

$$
\left(x_{31}, x_{30}, \ldots, x_{0}\right)^{t}=M_{\sigma_{1}^{-1}} \cdot\left(y_{31}, y_{30}, \ldots, y_{0}\right)^{t},
$$

where

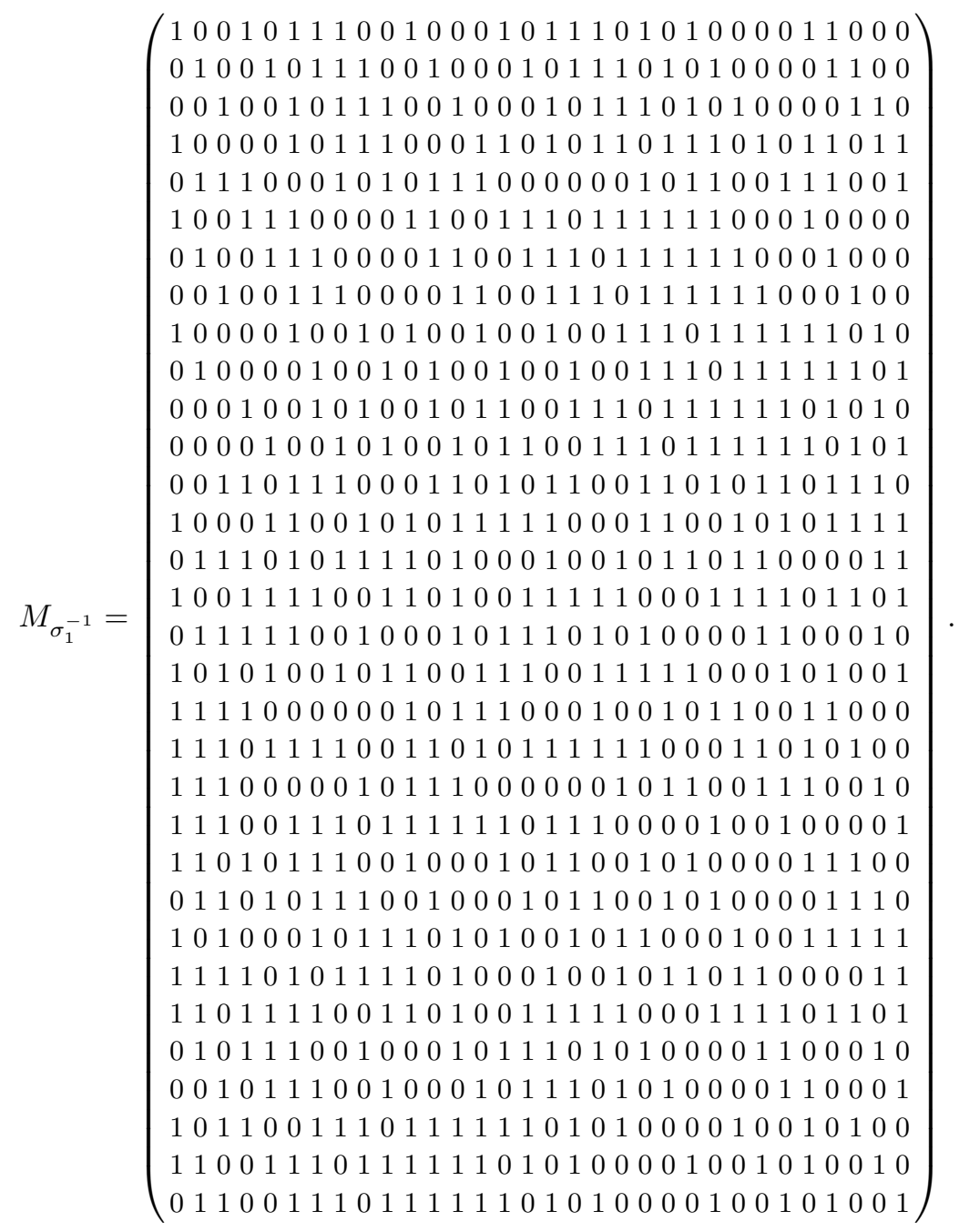

